\title{
Focos de dirofilariose canina na Ilha do Marajó: um fator de risco para a saúde humana
}

\author{
Focus of canine heartworm disease in Marajó Island, North of Brazil: \\ a risk factor for human health
}

\author{
Lourdes Maria Garcez ${ }^{1,2}$, Nazaré Fonseca de Souza ${ }^{3}$, Eduardo Ferreira Mota ${ }^{1,2}$, \\ Luís Antonio Jerônimo Dickson ${ }^{1,2}$, Wandercleyson Uchoa Abreu ${ }^{3}$, \\ Vânia de Fátima do Nascimento Cavalcanti ${ }^{3}$ e Patrick Abdala Fonseca Gomes ${ }^{1,2}$
}

\begin{abstract}
RESUMO
A ocorrência de dirofilariose pulmonar humana relaciona-se com a prevalência de infecção por Dirofilaria immitis na população canina. Várias espécies de mosquitos são vetores desse nematóide. Analisaram-se amostras de sangue canino coletados nas vilas Pingo d'Água e União, município de Salvaterra (Ilha do Marajó, PA), em junho, 2004 (n=34) e abril, 2005 (N=90). Os diagnósticos parasitológico e imunológico (ELISA - kit SNAP® $3 D X^{T M}$, Biobrasil) foram comparados $\left(\chi^{2}, \alpha=0,05\right)$ no exame de 34 amostras. A prevalência na população $(N=90)$ foi avaliada pelo ELISA. O ELISA revelou mais positivos $(25 / 34 ; 73,5 \%)$ que a gota espessa $(23 / 34,67,6 \%)$ e o Knott $(21 / 34,61,8 \%)$, mas a diferença não foi significativa $(p>0,05)$. A frequiência de infecção por D. immitis na faixa de 0 a 2 anos foi $58 \%$, enquanto em cães mais velhos foi 100\%. A prevalência da dirofilariose canina em Pingo d'Água e Vila União foi alta (53,5\%), indicando risco de transmissão do parasito às pessoas nessa área.
\end{abstract}

Palavras-chaves: Dirofilaria immitis. Prevalência. Cão. Dirofilariose pulmonar humana.

\begin{abstract}
The occurrence of human pulmonary dirofilariasis maintains a relation with the prevalence of Dirofilaria immitis infection in the canine population. Several mosquito species are vectors of this nematode. Canine blood samples collected in Pingo d'Água and União villages, Salvaterra municipality (Marajó Island, Pará), in June, 2004 (n=34) and April, 2005 (N=90) were analyzed. Parasitological and immunological (ELISA - kit SNAP® 3DXTM, Biobrasil) diagnoses were compared following the examination of 34 samples. The prevalence in the population ( $N=90)$ was evaluated by means of ELISA. ELISA revealed more positive samples $(25 / 34 ; 73.5 \%)$ than thick smears $(23 / 34,67.6 \%)$ or Knott $(21 / 34,61.8 \%)$, but the differences were not significant $(p>0.05)$. The frequency of $\mathrm{D}$. immitis infection was $58 \%$ in dogs ranging from 0-2 years old, whereas in older dogs it was 100\%. The prevalence of canine dirofilariasis was high in Pingo d'Água and Vila União (53.5\%), indicating the risk of parasite transmission to the people in this area.
\end{abstract}

Key-words: Dirofilaria immitis. Prevalence. Dog. Human pulmonary dirofilariasis.

A dirofilariose é uma zoonose de cães causada pelo nematóide filarídeo Dirofilaria immitis e transmitida por mais de 70 espécies dos gêneros Aedes, Anopheles e Culex ${ }^{1}$. os vermes adultos alojam-se no coração direito e suas microfilárias, formas infectantes ao vetor, encontram-se no sangue periférico. Embora o cão seja o hospedeiro natural mais importante, há riscos de transmissão ao homem. No homem o ciclo de vida do verme não se completa até a fase adulta e o parasita pode ser encontrado, ainda na forma larvar $\mathrm{L}_{4}$, em nódulo pulmonar, normalmente confundido com neoplasia ${ }^{7}$. Vários casos de dirofilariose pulmonar humana vêm sendo registrados no Brasil ${ }^{6} 781718$. Para a ocorrência

\footnotetext{
1. Seção de Parasitologia do Instituto Evandro Chagas da Secretaria de Vigilância em Saúde do Ministério da Saúde, Belém, PA. 2. Centro Universitário do Pará, Belém, PA. 3. Universidade Federal Rural da Amazônia, Belém, PA.

Auxílio financeiro: CESUPA e IEC/SVS/MS

Endereço para correspondência: Dra. Lourdes Maria Garcez. Seção de Parasitologia/SVS/IEC/MS. BR 316, Km 7, Bairro Levilândia, 67030-000 Ananindeua, PA. Tel: 5591 3214-2152; Fax: 5591 3214-2043.

e-mail: lourdesgarcez@iec.pa.gov.br

Recebido para publicação em 2/8/2005

Aceito em 15/5/2006
} 
dessa patologia emergente contribuem o tamanho elevado da população canina, prevalência da infecção em cães, densidade dos vetores e frequiência de exposição do homem às picadas desses insetos ${ }^{7}$. Os estudos realizados no Brasil relatam maior prevalência em áreas costeiras, mas a infecção existe também distante do litoral ${ }^{11}$. Nenhuma investigação sobre a prevalência da dirofilariose canina foi realizada anteriormente na Ilha do Marajó. O município de Salvaterra possui 158 comunidades e o total de 20.168 habitantes, de acordo com o Instituto Brasileiro de Geografia e Estatística (www.ibge.com.br). As vilas Pingo d'Água e União fazem parte de um complexo de 40 vilas cuja população canina é elevada e onde uma outra importante zoonose de cães, a leishmaniose visceral, está presente de forma endêmica, constituindo um problema de saúde importante para o município. Esse estudo relata a prevalência da infecção por $D$. immitis na população canina das duas vilas mencionadas e discute o risco de transmissão ao homem.

\section{MATERIAL E MÉTODOS}

0 protocolo de pesquisa foi avaliado e aprovado pelo Comitê de Ética em Pesquisa com Animais do Instituto Evandro Chagas, em Belém, PA (Processo 0015/2004/CEPAN/ IEC/SVS/MS). Os dados populacionais não foram coletados como atividade desta pesquisa, mas disponibilizados pelo banco de dados de outra concomitante, centrada no controle da leishmaniose visceral humana e canina no município de Salvaterra, também coordenada pela autora principal deste estudo e aprovada no Comitê de Ética em Pesquisa do Instituto Evandro Chagas (Processo 03/2004/CEP/IEC/SVS/MS).

Área de estudo. As vilas Pingo d'Água e União encontramse a nordeste da sede do município de Salvaterra, da qual distanciam-se 13,5 e $11,5 \mathrm{~km}$, respectivamente. As vilas são adjacentes e distantes, em linha reta, $6 \mathrm{~km}$ da orla marítima. 0 município (Latitude - $00^{\circ} 45^{\prime} 12^{\prime \prime}$; Longitude 48 31'00") situase na Ilha do Marajó, no Estado do Pará. Extensas áreas de desmatamento estão presentes aos arredores das vilas.

Diagnóstico e freqüência da infecção canina. Utilizou-se 34 cães das duas vilas para um inquérito hemoscópico, por meio da gota espessa ${ }^{10}$ e Knott modificado ${ }^{16}$, e imunológico, por meio do Ensaio imunoenzimático (kit SNAP ${ }^{3}$ 3DX ${ }^{\mathrm{TM}}$ - Biobrasil, BR) para evidência sorológica de antígenos do verme adulto ${ }^{24}$, comparando-se os métodos $\left(\chi^{2}, \alpha=0,05\right)$. A amostra foi obtida em junho de $2004 \mathrm{com} o$ objetivo de aplicação e comparação dos métodos de diagnóstico. Foi composta de cães domésticos, sem raça definida, com idades variando de 6 meses a 14 anos, conforme informado pelos donos. 0 kit SNAP ${ }^{\circledR}$ 3-DX ${ }^{\mathrm{TM}}$ é um teste rápido com leitura visual que se utiliza da tecnologia ELISA. Três gotas do soro e 4 do conjugado foram dispensadas no dispositivo que detecta antígenos do verme adulto da espécie $D$. immitis. Decorridos 8 minutos, reações positivas eram reveladas pelo surgimento de área azul circular, à semelhança do controle positivo, e reações negativas pela ausência de cor. Realizou-se o screenning test para o ELISA com uso do kit SNAP $® 3-\mathrm{DX}^{\mathrm{TM}}$ comparando-o ao exame parasitológico (gota espessa e Knott modificado), onde: $\mathrm{a}=$ verdadeiros positivos, $\mathrm{b}=$ falsos positivos, $\mathrm{c}=$ falsos negativos e $\mathrm{d}=$ verdadeiros negativos. Foram calculados a sensibilidade $(a / a+c \times 100)$, especificidade $(d / b+d \times 100)$ e os valores de predição positivo $(a / a+b \times 100)$ e negativo $(d / c+d \times 100)$. Classificou-se os cães de acordo com idade e freqüência de infecção por $D$. immitis.

População canina. Para um segundo levantamento, em abril de 2005, realizou-se censo da população canina nas duas vilas. A proporção da população canina em relação à humana (dados pré-existentes) foi calculada.

Determinação da prevalência. Obteve-se sangue de todos os cães encontrados durante o censo $(\mathrm{N}=90)$ nas duas comunidades, em abril de 2005. Os soros foram testados somente pelo ELISA com o kit SNAP ${ }^{\circledR} 3 \mathrm{DX}^{\mathrm{TM}}$. 0 percentual de positivos correspondeu à prevalência.

\section{RESULTADOS}

A freqüência de positivos na amostra foi alta com qualquer um dos métodos utilizados. Embora o ELISA tenha revelado maior número de positivos $(25 / 34 ; 73,5 \%)$ em relação à gota espessa $(23 / 34,67,6 \%)$ e ao Knott $(21 / 34,61,8 \%)$, a diferença não foi significativa $(\mathrm{p}>0,05)$. Não se investigou a carga parasitária, mas o achado de microfilárias abundantes foi freqüente ao exame microscópico pela gota espessa. Houve alta concordância entre resultados positivos e negativos quando comparados os métodos parasitológico e imunológico, exceto por um único cão que teve diagnóstico positivo pelo ELISA, mas negativo ao exame parasitológico (1/34, 2,9\%). Considerando os três métodos em conjunto 26/34 (76,5\%) cães foram positivos (Figura 1), incluindo todos acima de 2 anos (15/34). Cães negativos para D. immitis (11/34) foram encontrados somente na faixa de 0 a 2 anos (Tabela 1 ).

Os índices de desempenho do ELISA com o kit SNAP® 3DX ${ }^{\mathrm{TM}}$ para o diagnóstico da infecção canina, resultado da

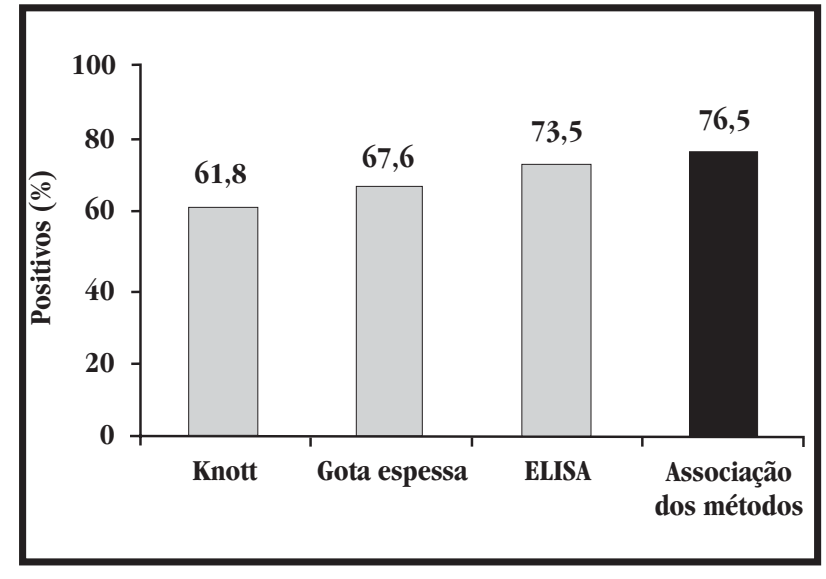

Figura 1 - Diagnóstico da dirofilariose em cães das vilas Pingo d'Água e União, município de Salvaterra, Pará (junho/2004). Pesquisa de microfilárias no sangue por meio do Knott modificado e gota espessa e de antígenos do verme adulto no soro pelo ELISA - kit SNAP@ $3 D X^{T M}(P>0,05)$. 
Tabela 1 - Dirofilariose canina por faixa etária nas vilas Pingo d'Água e União, município de Salvaterra, Estado do Pará (junho/2004).

\begin{tabular}{|c|c|c|c|c|c|c|}
\hline \multirow[b]{2}{*}{ Idades (anos) ${ }^{\mathrm{a}}$} & \multicolumn{2}{|c|}{ Cães examinados } & \multicolumn{2}{|c|}{ Positivos } & \multicolumn{2}{|c|}{ Negativos } \\
\hline & $\begin{array}{c}\mathrm{n}^{0} \\
(\mathrm{M}: \mathrm{F})^{\mathrm{b}}\end{array}$ & $\%$ & $\begin{array}{c}\mathrm{n}^{0} \\
(\mathrm{M}: \mathrm{F})\end{array}$ & $\%$ & $\begin{array}{c}\mathrm{n}^{0} \\
(\mathrm{M}: \mathrm{F})\end{array}$ & $\%$ \\
\hline $0 \mid-2$ & $\begin{array}{c}19 \\
(10: 9)\end{array}$ & 56,0 & $\begin{array}{c}11 \\
(6: 5)\end{array}$ & 32,4 & $\begin{array}{c}8 \\
(4: 4)\end{array}$ & 23,5 \\
\hline $2 \mid-4$ & $\begin{array}{c}7 \\
(2: 5)\end{array}$ & 20,0 & $\begin{array}{c}7 \\
(2: 5)\end{array}$ & 20,6 & 0 & 0,0 \\
\hline $4 \mid-6$ & $\begin{array}{c}4 \\
(3: 1)\end{array}$ & 12,0 & $\begin{array}{c}4 \\
(3: 1)\end{array}$ & 11,8 & 0 & 0,0 \\
\hline $6 \mid-14$ & $\begin{array}{c}4 \\
(1: 3) \\
\end{array}$ & 12,0 & $\begin{array}{c}4 \\
(1: 3)\end{array}$ & 11,8 & 0 & 0,0 \\
\hline Total & $\begin{array}{c}34 \\
(16: 18)\end{array}$ & 100,0 & $\begin{array}{c}26 \\
(12: 14)\end{array}$ & 76,5 & $\begin{array}{c}8 \\
(4: 4)\end{array}$ & 23,5 \\
\hline
\end{tabular}

${ }^{\mathrm{a}}$ informado pelos donos dos cães; ${ }^{\mathrm{b}} \mathrm{M}=$ machos, $\mathrm{F}=$ fêmeas

comparação com o exame parasitológico na amostra, foram os seguintes: sensibilidade (96\%), especificidade (82\%) e valores de predição positivo (92\%) e negativo (90\%).

A população das vilas Pingo d'Água e União era de 485 pessoas (Pingo d'Água: 170; União: 315) na ocasião. 0 censo da população canina realizado nesse estudo revelou a existência de 105 cães, todos sem raça definida. Não havia cães vadios, embora transitassem livremente nas duas vilas. Todos tinham donos e foram identificados pelo nome. A população canina corresponde a $22 \%$ da humana. A razão entre o número de pessoas e de cães foi igual a 4,6, existindo um cão para cerca de quatro habitantes. Quando realizada a coleta de sangue da população canina encontraram-se apenas 90 dos 105 cães. Quinze cães mencionados como existentes pelos moradores do local não estavam presentes nas três ocasiões que se realizou a coleta de sangue, ao longo de uma mesma semana, por estarem acompanhando seus donos em atividades de caça ou por terem se afastado muito da residência no momento da coleta. Tomando-se esta população $(\mathrm{N}=90)$, constatou-se positividade ao ELISA em 48 animais, o que indica prevalência de $53,5 \%$ de infecção por $D$. immitis.

\section{DISCUSSÃO}

A comparação dos métodos de diagnóstico na amostra de cães das vilas Pingo d'Água e União, município de Salvaterra, Estado do Pará, revela diferença não significante entre os resultados (Figura 1), apesar da sensibilidade da sorologia para detecção de antígenos de $D$. immitis ser considerada superior a do exame parasitoscópico ${ }^{22}$. Embora não tenha sido estabelecida a carga parasitária, a elevada freqüência de cães com microfilaremia observada nesse estudo, especialmente em amostras de animais com mais de dois anos (Tabela 1), e a facilidade com que microfilárias eram encontradas ao exame microscópico sugerem que a alta sensibilidade dos métodos parasitológicos poderia estar relacionada com elevada carga parasitária nos cães daquela área, o que ainda precisa ser investigado.

A idade do cão é um fator de risco importante determinado pelo tempo de exposição na área endêmica ${ }^{319} \mathrm{e}$ a prevalência de animais com microfilárias de $D$. immitis aumenta progressivamente com a faixa etária ${ }^{2021}$. Microfilárias não foram encontradas em cães com menos de um ano em estudo conduzido na Cidade de São Luis, mas o índice de positividade foi alto para aqueles entre dois a seis anos de idade $(86,4 \%)$ e acima de nove anos $(100 \%)^{2}$. Em Belém, o índice de positividade para microfilárias foi também mais elevado entre cães de dois a quatro anos ${ }^{20}$. No caso das vilas Pingo d'Água e União, 100\% dos cães da amostra examinada com idade superior a dois anos estavam com microfilaremia (Tabela 1) ${ }^{14}$. Essas observações indicam que as duas vilas constituem focos de transmissão intensa de D. immitis entre a população canina.

Várias espécies de mosquitos são vetores potenciais de D. immitis $^{1}$. Os programas de controle, dirigidos contra enfermidades transmitidas por insetos, como dengue e malária, exercem pressão sobre a população de vetores, o que contribuiu com o declínio na prevalência nacional da dirofilariose canina no Brasil, de 7,9\% em 1988 para 2\% em $2001^{12}$. Entretanto, em alguns locais esses programas podem não ser tão efetivos.

A dirofilariose canina está presente em vários estados de todas as regiões do Brasil. As maiores prevalências descritas no país estão em áreas banhadas por rios e mares, providas ou próximas de lagos ${ }^{7}$. Na orla marítima de São Luis, foco ativo de transmissão da $D$. immitis é descrita a prevalência de $24 \%$, com destaque aos bairros Olho d'Água (46\%), Alto do Calhau (43\%) e Calhau (25\%) $)^{2}$. Nos distritos do litoral em Maceió, Estado do Alagoas, a prevalência de filariose canina $(3,7 \%)$ é superior a observada no centro da cidade, em áreas mais urbanizadas $(1,2 \%)^{5}$. Na Cidade de Florianópolis, Estado do Paraná, varia ao longo da costa marítima de 0 a $20 \%{ }^{4}$.

No município de Salvaterra, localizado em zona costeira da Ilha do Marajó, Estado do Pará, não há relatos anteriores sobre dirofilariose canina. A prevalência descrita neste estudo para a população de cães das vilas Pingo d'Água e União (53,5\%) é seguramente uma das maiores já relatadas no Brasil. As razões desse alto índice devem estar relacionadas às características hidrográficas da área, ocupação desordenada do ambiente, desmatamento e fatores sócio-econômicos. Contudo, considerando tratar-se de uma área amazônica, onde não há estudos sobre o tema, outras influências ambientais, muito distintas daquelas vigentes nos diversos locais do Brasil onde a endemia está presente, poderiam também estar contribuindo para a alta prevalência observada.

Casos de dirofilariose pulmonar humana têm sido diagnosticados em diferentes regiões do Brasil, em adultos entre 40 e 60 anos, e já ultrapassam 50 em todo o país ${ }^{6781718}$, caracterizando uma doença emergente. As lesões são normalmente diagnosticadas em exames de rotina e podem ser confundidas com neoplasias pulmonares. Pouco mais de $50 \%$ dos pacientes apresentam sintomas decorrentes da presença do nódulo pulmonar. Febre, tosse, dor no peito, infecções pulmonares e hemoptise são alguns dos mais freqüentes. A importância em saúde pública, contudo, não está associada à doença clínica que a $D$. immitis produz em humanos, mas à gravidade das doenças que os achados 
radiográficos sugerem existir, o que requer um extenso trabalho de acompanhamento clínico culminando freqüentemente com toracotomia ${ }^{1722}$. Considerando que a ocorrência dessa enfermidade guarda relação com a alta prevalência de infecção na população canina ${ }^{7}$, uma busca ativa em indivíduos daquela área poderia revelar novos casos da doença humana.

Os resultados aqui obtidos permitem especular a existência de outros focos de dirofilariose canina em Salvaterra, reforçando a importância da determinação da prevalência em todo o município, o que poderia ser investigado com uso do kit SNAP ${ }^{3}$ 3-DX ${ }^{\mathrm{TM}}$, que se mostrou sensível (96\%) e específico (82\%) nesse estudo. Não obstante, uma investigação clínica e laboratorial na população humana, com uso de diagnóstico por imagem, conduzida em indivíduos residentes naquelas vilas, ou em outras onde se constate alta prevalência de $D$. immitis em cães, poderia revelar casos de dirofilariose pulmonar. Um teste sorológico para identificação de casos humanos não está comercialmente disponível, mas um ELISA com esse propósito é passível de ser desenvolvido e seria útil à identificação precoce de infecção subclínica em áreas endêmicas ${ }^{12} 21$.

A epidemiologia da dirofilariose, incluindo uma investigação sobre espécies de insetos vetores, necessita ser esclarecida no município de Salvaterra sob a ótica de controle da infecção canina e prevenção da doença humana.

\section{AGRADECIMENTOS}

Os autores agradecem ao Instituto Evandro Chagas, Centro Universitário do Pará e Prefeitura de Salvaterra pelo apoio logístico e suporte financeiro; aos Drs. Marinete Póvoa, Orin Courtenay e Samuel Price pelas facilidades proporcionadas ao trabalho de campo; à Dra. Joana Favacho, aos estudantes Joyce Cardoso e Hugo Favacho e à técnica Rita Martins pelo apoio na execução do trabalho de campo.

\section{REFERÊNCIAS BIBLIOGRÁFICAS}

1. Ahid SMM, Lourenço-de-Oliveira R. Mosquitos vetores potenciais de dirofilariose canina na Região Nordeste do Brasil. Revista de Saúde Pública 33:560-565, 1999

2. Ahid SMM, Lourenço-de-Oliveira R, Saraiva LQ. Dirofilariose canina na Ilha de São Luís, Nordeste do Brasil: uma zoonose potencial. Cadernos de Saúde Pública 15:405-412,1999.

3. Almeida MAO, Barros MTG, Santos EP, Ayres MCC, Guimarães JE, Gondim FP. Parasitismo de cães por microfilárias de Dirofilaria immitis: influência da raça, sexo e idade. Revista Brasileira de Saúde e Produção Animal 2:5964, 2001.

4. Araujo RT, Marcondes CB, Bastos LC, Sartor DC. Canine dirofilariasis in the region of Conceição Lagoon, Florianópolis, and in the Military Police kennel, São José, State of Santa Catarina, Brazil. Veterinary Parasitology 113:239-242, 2003.
5. Brito AC, Vila-Nova MC, Rocha DAM, Costa LG, Viana LS, Lopes Jr RR, Fontes G, Rocha EMM, Regis L. Prevalência da dirofilariose canina causada por Dirofilaria immitis e Dipetalonema reconditum em Maceió, Alagoas, Brasil. Cadernos de Saúde Pública 17:1497-1504, 2001

6. Campos JRM, Barbas CS, Filomeno LT, Fernandez A, Minamoto H, Filho JV, Jatene FB. Human pulmonary dirofilariasis: analysis of 24 cases from São Paulo, Brazil. Chest 112:729-733, 1997.

7. Cavallazzi RS, Cavallazzi AC, Souza IV, Cardoso JJD. Dirofilariose pulmonar humana: relato de sete casos. Jornal de Pneumologia 28:100-102, 2002.

8. Ciferri F. Human pulmonary dirofilariasis in the United States: a critical review. American Journal of Tropical Medicine and Hygiene 31:302-308, 1982

9. Hendrix CM, Brunner CJ, Bellamy LK. Natural transmission of Dirofilaria immitis by Aedes aegypti. Journal of American Mosquito Control Associassion 2:48-51, 1986.

10. Knight DH. Heartworm heart disease. Advances of Veterinary Sciences and Comparative Medicine 21:107-149, 1977.

11. Labarthe N, Almosny N, Guerrero J, Duque-Araujo AM. Description of the occurrence of canine dirofilariasis in the State of Rio de Janeiro, Brazil. Memórias do Instituto Oswaldo Cruz 92:47-51, 1997.

12. Labarthe N, Guerrero J. Epidemiology of heartworm: What is hapenning in South America and Mexico? Veterinary Parasitology 133:149-156, 2005.

13. Mori S, Mizooguchi A, Kawabata M, Nakamura-Uchiyama F, Nawa Y, Osame M. Pulmonary dirofilariasis with serologic study on familiary infection with Dirofilaria immitis. Internal Medicine 43:327-330, 2005.

14. Mota EF, Dickson LA, Souza NF, Gomes PAF, Garcez LM. Dirofilariose canina na Ilha do Marajó. In: Resumos do XLI Congresso da Sociedade Brasileira de Medicina Tropical, Brasília, p. 473, 2005.

15. Nelson CT, McCall JW, Rubin SB, Buzhardt LF, Dorion DW, Graham W, Longhofer SL, Guerrero J, Robertson-Plouch C, Paul A, Executive Board of the American Hearthworm Society. 2005 Guidelines for the diagnosis, prevention and management of heartworm (Dirofilaria immitis) infection in dogs. Veterinary Parasitology 133:255-266, 2005

16. Newton WL, Wright WH. The occurrence of a dog filariid other than Dirofilaria immitis in the United States. Journal of Parasitology 42:246-258, 1956.

17. Ro JY, Tsakalakis PJ, White VA, Luna MA, Chang-Tung EG, Green L, Cribbett L, Ayala AG. Pulmonary dirofilariasis: the great imitator of primary or metastatic lung tumor. A clinicopathologic analysis of seven cases and a review of the literature. Human Pathology 20:69-76, 1989.

18. Rodrigues-Silva R, Guerra RJ, Almeida FB, Machado Silva JR, Paiva DD. Human pulmonary dirofilariasis at Rio de Janeiro, Brazil: a case report. Revista da Sociedade Brasileira de Medicina Tropical 37:56-59, 2004.

19. Selby LA, Corwin RM, Hayes Jr HM. Risk factors associated with canine heartworm infection. Journal of American Veterinarian Medical Association. 176:33-35, 1980 .

20. Souza NF, Benigno RNM, Figueiredo SK, Silva D, Gonçalves R, Peixoto PC, Serra-Freire NM. Prevalência de Dirofilaria immitis em cães no município de Belém, PA, com base na microfilaremia. Revista Brasileira de Parasitologia Veterinária 6:83-86, 1997.

21. Souza NF, Larsson MHMA. Freqüência de dirofilariose canina (D. immitis) em algumas regiões do Estado de São Paulo por meio da detecção de antígenos circulantes. Arquivo Brasileiro de Medicina Veterinária e Zootecnia 53:321-325, 2001.

22. Theis, JH. Public health aspects of dirofilariasis in the United States. Veterinary Parasitology 133: 157-180, 2005.

23. Thilsted JP, Whorton J, Hibbs CM, Jillson GP, Steece R, Stromei M. Comparison of four serotests for the detection of Dirofilaria immitis infection in dogs. American Journal of Veterinarian Research 48:837-841,1987.

24. Weil GJ. Dirofilaria immitis: identification and partial characterization of parasite antigens in the serum of infected dogs. Experimental Parasitology 64:244-251, 1987 